\title{
Asymptotics of joint maxima for discontinuous random variables
}

\section{Journal Article}

\section{Author(s):}

Feidt, A.; Genest, Chr.; Neslehova, J.

Publication date:

2010-03

Permanent link:

https://doi.org/10.3929/ethz-b-000020735

Rights / license:

In Copyright - Non-Commercial Use Permitted

Originally published in:

Extremes 13(1), https://doi.org/10.1007/s10687-009-0085-7 


\title{
Asymptotics of joint maxima for discontinuous random variables
}

\author{
A. Feidt • Chr. Genest • J. Nešlehová
}

Received: 23 April 2008 / Revised: 22 December 2008 /

Accepted: 2 April 2009 / Published online: 25 April 2009

(C) Springer Science + Business Media, LLC 2009

\begin{abstract}
This paper explores the joint extreme-value behavior of discontinuous random variables. It is shown that as in the continuous case, the latter is characterized by the weak limit of the normalized componentwise maxima and the convergence of any compatible copula. Illustrations are provided and an extension to the case of triangular arrays is considered which sheds new light on recent work of Coles and Pauli (Stat Probab Lett 54:373-379, 2001) and Mitov and Nadarajah (Extremes 8:357-370, 2005). This leads to considerations on the meaning of the bivariate upper tail dependence coefficient of Joe (Comput Stat Data Anal 16:279-297, 1993) in the discontinuous case.
\end{abstract}

Keywords Copula - Discrete distribution - Joint extremes • Maximum • Upper tail dependence $\cdot$ Triangular array

AMS 2000 Subject Classifications $60 \mathrm{G} 70 \cdot 62 \mathrm{E} 20$

\footnotetext{
A. Feidt

Institut für Mathematik, Universität Zürich, Winterthurerstraße 190, 8057 Zürich, Switzerland

e-mail: anne.feidt@math.uzh.ch

C. Genest

Département de mathématiques et de statistique, Université Laval, 1045, avenue de la Médecine, Québec,

Québec, Canada G1V OA6

e-mail: Christian.Genest@mat.ulaval.ca

J. Nešlehová $(\varangle)$

Departement Mathematik, ETH Zürich, Rämistraße 101, 8092 Zürich, Switzerland

e-mail: johanna.neslehova@math.ethz.ch
} 


\section{Introduction}

Let $\left(X_{1}, \ldots, X_{d}\right)$ be a random vector with cumulative distribution function $H$ and for each $j \in\{1, \ldots, d\}$ and $x \in \mathbb{R}$, let $F_{j}(x)=\mathrm{P}\left(X_{j} \leqslant x\right)$. Given independent copies $\left(X_{11}, \ldots, X_{1 d}\right), \ldots,\left(X_{n 1}, \ldots, X_{n d}\right)$ of the random vector $\left(X_{1}, \ldots, X_{d}\right)$, write

$$
M_{n j}=\max \left(X_{1 j}, \ldots, X_{n j}\right)
$$

for every $j \in\{1, \ldots, d\}$. Then $H$ is said to belong to the domain of attraction of a non-degenerate extreme-value distribution $H^{*}$, denoted $H \in \mathscr{M}\left(H^{*}\right)$, if there exist constants $a_{n j}, b_{n j} \in \mathbb{R}$ with $b_{n j}>0$ such that for all $x_{1}, \ldots, x_{d} \in \mathbb{R}$,

$$
\lim _{n \rightarrow \infty} \mathrm{P}\left(\frac{M_{n 1}-a_{n 1}}{b_{n 1}} \leqslant x_{1}, \ldots, \frac{M_{n d}-a_{n d}}{b_{n d}} \leqslant x_{d}\right)=H^{*}\left(x_{1}, \ldots, x_{d}\right) .
$$

In particular,

$$
F_{j}^{*}(x)=\lim _{n \rightarrow \infty} \mathrm{P}\left(\frac{M_{n j}-a_{n j}}{b_{n j}} \leqslant x\right)
$$

then belongs to the class of univariate extreme-value distributions characterized by Fisher and Tippett (1928). The constants $a_{n j}$ and $b_{n j}$ needed for convergence in Eq. 2 are provided by univariate extreme-value theory.

Convergence of the margins is not sufficient to ensure that Eq. 2 holds, however. Conditions on the joint distribution are needed; they may be found, e.g., in the books by Resnick (1987) and Beirlant et al. (2004), or in the survey paper by Fougères (2004). When the marginal distributions are continuous, the condition can be expressed simply in terms of the unique copula associated with $H$, i.e., the function $C$ such that

$$
H\left(x_{1}, \ldots, x_{d}\right)=C\left\{F_{1}\left(x_{1}\right), \ldots, F_{d}\left(x_{d}\right)\right\}
$$

for all $x_{1}, \ldots, x_{d} \in \mathbb{R}$. As shown, e.g., by Galambos (1987, Theorem 5.2.3), Eq. 2 holds if and only if Eq. 3 is verified for every $j \in\{1, \ldots, d\}$ and if

$$
\lim _{t \rightarrow \infty} C^{t}\left(u_{1}^{1 / t}, \ldots, u_{d}^{1 / t}\right)=C^{*}\left(u_{1}, \ldots, u_{d}\right),
$$

for all $u_{1}, \ldots, u_{d} \in[0,1]$, where $C^{*}$ is the copula associated with $H^{*}$. As observed, e.g., by McNeil et al. (2005, Section 7.5.3), this is equivalent to saying that for all $x_{1}, \ldots, x_{d} \geqslant 0$, one has

$$
\lim _{s \rightarrow 0} \frac{1-C\left(1-s x_{1}, \ldots, 1-s x_{d}\right)}{s}=-\log \left\{C^{*}\left(e^{-x_{1}}, \ldots, e^{-x_{d}}\right)\right\} .
$$

In practice, however, there are situations where the variables of interest are discontinuous. In principle, these cases are covered by the existing theory but as illustrated in Section 2, the conditions under which marginal distributions have a non-degenerate extreme-value behavior are often not met. This led Anderson et al. (1997) to consider triangular arrays, i.e., situations where $M_{n j}$ is still defined as in Eq. 1 but for each integer $n \geqslant 1$, the sample 
$\left(X_{11: n}, \ldots, X_{1 d: n}\right), \ldots,\left(X_{n 1: n}, \ldots, X_{n d: n}\right)$ comes from a distribution $H_{n}$ with margins $F_{n}$ and $G_{n}$. This framework was also adopted, e.g., by Nadarajah and Mitov (2002, 2004) and Mitov et al. (2003).

An additional difficulty associated with discontinuous margins is that Eq. 4 does not hold for a single $C$, but for an infinite collection of such functions, not all of which are copulas; see, e.g., Genest and Nešlehová (2007). In the context of extremes, this unidentifiability issue was explicitly recognized by Deheuvels (1978) and later accounted for by Galambos (1987) and Hsing (1989). Their results are summarized and complemented in Section 3.

An extension to triangular arrays is then presented in Section 4, which also sheds new light on recent work of Coles and Pauli (2001) and Mitov and Nadarajah (2005). Finally, Section 5 investigates properties of the bivariate upper tail dependence coefficient of Joe (1993) for discontinuous random variables. Concluding comments are made in Section 6 and mathematical derivations are grouped in a series of Appendices.

\section{Extremal behavior of univariate discontinuous distributions}

Let $X$ be a random variable with distribution $F$ and survival $\bar{F}$. When $X$ is absolutely continuous, denote its density by $f$ and let $\lambda=f / \bar{F}$ be the corresponding hazard rate. Because $F$ may have jumps, it will be useful to consider its left-continuous version,

$$
F_{\rightarrow}(x)=\lim _{h \downarrow 0} F(x-h)
$$

for all $x \in \mathbb{R}$. Finally, $x_{F}=\sup \{x: F(x)<1\}$ denotes the right endpoint of $F$.

Let $X_{1}, \ldots, X_{n}$ be independent copies of $X$ and write $M_{n}=$ $\max \left(X_{1}, \ldots, X_{n}\right)$. Then $F$ is said to belong to the domain of attraction of a non-degenerate distribution $F^{*}$ if there exist constants $a_{n}, b_{n} \in \mathbb{R}$ with $b_{n}>0$ such that $\left(M_{n}-a_{n}\right) / b_{n}$ converges in law to $F^{*}$. As shown by Leadbetter et al. (1983), this can only occur if

$$
\lim _{x \rightarrow x_{F}} \frac{\bar{F}(x)}{1-F_{\rightarrow}(x)}=1 .
$$

Thus if $x_{F}<\infty$ and $F_{\rightarrow}\left(x_{F}\right)<F\left(x_{F}\right)$ as for the binomial distribution, then

$$
\lim _{x \rightarrow x_{F}} \frac{\bar{F}(x)}{1-F_{\rightarrow}(x)}=0 .
$$

Hence the limiting behavior of $F$ is then degenerate. Embrechts et al. (1997, Section 3.1) show that Eq. 7 also fails for the Poisson, geometric and negative binomial distributions. Nonetheless, there are many examples of discrete random variables that have a non-degenerate extreme-value behavior.

Example 1 Let $X \geqslant 0$ be an absolutely continuous random variable with $x_{F}=\infty$. Denote by $\lfloor x\rfloor$ the integer part of $x \in \mathbb{R}$ and let $\lceil x\rceil$ stand for 
the smallest integer $n$ such that $n \geqslant x$. Consider the integer-valued random variable $\lceil X\rceil$ with distribution function $\lceil F\rceil(x)=F(\lfloor x\rfloor)$ for all $x \geqslant 0$. Observe that since $\bar{F}$ is decreasing,

$$
0 \leqslant \frac{P(\lceil X\rceil=n)}{1-\lceil F\rceil(n-1)}=\frac{F(n)-F(n-1)}{\bar{F}(n-1)}=\int_{n-1}^{n} \lambda(u) \frac{\bar{F}(u)}{\bar{F}(n-1)} \mathrm{d} u \leqslant \int_{n-1}^{n} \lambda(u) \mathrm{d} u .
$$

Thus if $\lambda(x) \rightarrow 0$ as $x \rightarrow \infty$, the right-hand side tends to zero as $n \rightarrow \infty$. Therefore,

$$
\lim _{n \rightarrow \infty} \frac{1-\lceil F\rceil(n)}{1-\lceil F\rceil(n-1)}=1-\lim _{n \rightarrow \infty} \frac{P(\lceil X\rceil=n)}{1-\lceil F\rceil(n-1)}=1 .
$$

In other words, $\lceil X\rceil$ satisfies Eq. 7. Furthermore, note that for $x \in \mathbb{R}_{+}$,

$$
1 \leqslant \frac{1-\lceil F\rceil(x)}{1-F(x)} \leqslant \frac{1-F(\lfloor x\rfloor)}{1-F(\lfloor x\rfloor+1)}=\exp \left(\int_{\lfloor x\rfloor}^{\lfloor x\rfloor+1} \lambda(t) \mathrm{d} t\right),
$$

because $\bar{F}(x)=\exp \left(-\int_{0}^{x} \lambda(t) \mathrm{d} t\right)$ for non-negative random variables. Thus the same condition on $\lambda$ ensures that $\{1-\lceil F\rceil(x)\} /\{1-F(x)\} \rightarrow 1$ as $x \rightarrow \infty$, i.e., $X$ and $\lceil X\rceil$ are tail equivalent. In particular, therefore, if $F$ is in the domain of attraction of either the Fréchet or the Gumbel distribution, the same applies to $\lceil F\rceil$.

Distributions for which $\lambda(x) \rightarrow 0$ as $x \rightarrow \infty$ include:

(i) The Pareto distribution $\mathscr{P}(\theta, \alpha)$ with parameters $\theta, \alpha>0$, distribution function $F(x)=1-(x / \theta)^{-\alpha}$ and hazard rate $\lambda(x)=\alpha / x$ for $x \geqslant \theta$.

(ii) The Weibull distribution $\mathscr{W}(\mu, c)$ with parameters $\mu>0$ and $c \in(0,1)$, distribution function $F(x)=1-\exp \left(-\mu x^{c}\right)$ and hazard rate $\lambda(x)=$ $\mu c x^{c-1}$ for $x>0$.

\section{Extremal behavior of vectors of discrete random variables}

Consider a random vector $\left(X_{1}, \ldots, X_{d}\right)$ from distribution $H$ with margins $F_{1}, \ldots, F_{d}$. Let $\mathscr{C}(H)$ be the class of copulas $C$ for which Eq. 4 holds for all $x_{1}, \ldots, x_{d} \in \mathbb{R}$. As mentioned, e.g., in Section 2.10 of Nelsen (2006), the members of $\mathscr{C}(H)$ coincide with $H\left\{F_{1}^{-1}\left(u_{1}\right), \ldots, F_{d}^{-1}\left(u_{d}\right)\right\}$ for all $\left(u_{1}, \ldots, u_{d}\right) \in$ $\operatorname{Ran}\left(F_{1}\right) \times \cdots \times \operatorname{Ran}\left(F_{d}\right)$. Here,

$$
\begin{aligned}
F_{j}^{-1}(u) & =\inf \left\{x \in \mathbb{R}: F_{j}(x) \geqslant u\right\}, \\
\operatorname{Ran}\left(F_{j}\right) & =\left\{u \in[0,1]: u=F_{j}(x) \text { for some } x \in \mathbb{R}\right\},
\end{aligned}
$$

for all $j \in\{1, \ldots, d\}$. Thus if the marginal distributions are continuous, $\mathscr{C}(H)$ reduces to a singleton, i.e., the copula $C$ in Eq. 4 is unique.

In all other cases, however, the class $\mathscr{C}(H)$ of compatible copulas is infinitely large and its members may differ considerably (Genest and Nešlehová 2007). As pointed out, e.g., by Marshall (1996), it is then more difficult to characterize weak convergence in terms of copulas. The following result, proved in Appendix 1, clarifies the issue. 
Proposition 1 Consider a sequence $\left(X_{11}, \ldots, X_{1 d}\right),\left(X_{21}, \ldots, X_{2 d}\right), \ldots$ of $m u$ tually independent random vectors such that $\left(X_{n 1}, \ldots, X_{n d}\right)$ has distribution function $H_{n}$ with margins $F_{n 1}, \ldots, F_{n d}$. Let $\left(X_{1}, \ldots, X_{d}\right)$ be another random vector with distribution function $H$ and margins $F_{1}, \ldots, F_{d}$. The following statements are equivalent:

(a) $\left(X_{n 1}, \ldots, X_{n d}\right) \rightsquigarrow\left(X_{1}, \ldots, X_{d}\right)$ as $n \rightarrow \infty$.

(b) $X_{n j} \rightsquigarrow X_{j}$ as $n \rightarrow \infty$ for $j \in\{1, \ldots, d\}$, and there exists $C \in \mathscr{C}(H)$ and a sequence $\left(C_{n}\right)$ such that $C_{n} \in \mathscr{C}\left(H_{n}\right)$, and $C_{n} \rightarrow C$ on $\operatorname{Ran}\left(F_{1}\right) \times \cdots \times$ $\operatorname{Ran}\left(F_{d}\right)$ as $n \rightarrow \infty$.

(c) $X_{n j} \rightsquigarrow X_{j}$ as $n \rightarrow \infty$ for $j \in\{1, \ldots, d\}$, and for all choices of $C_{n} \in$ $\mathscr{C}\left(H_{n}\right)$ and $C \in \mathscr{C}(H), C_{n} \rightarrow C$ uniformly on $\overline{\operatorname{Ran}\left(F_{1}\right)} \times \cdots \times \overline{\operatorname{Ran}\left(F_{d}\right)}$ as $n \rightarrow \infty$.

Now suppose that $\left(X_{11}, \ldots, X_{1 d}\right), \ldots,\left(X_{n 1}, \ldots, X_{n d}\right)$ are independent copies of $\left(X_{1}, \ldots, X_{d}\right)$. For fixed $j \in\{1, \ldots, d\}$, let $M_{n j}$ be the componentwise maximum defined in Eq. 1, whose joint distribution is $H_{n}\left(x_{1}, \ldots, x_{d}\right)=$ $H^{n}\left(x_{1}, \ldots, x_{d}\right)$ for all $x_{1}, \ldots, x_{d} \in \mathbb{R}$. Observe that if $C \in \mathscr{C}(H)$, then

$$
D_{n}\left(u_{1}, \ldots, u_{d}\right)=C^{n}\left(u_{1}^{1 / n}, \ldots, u_{d}^{1 / n}\right)
$$

is a copula in $\mathscr{C}\left(H_{n}\right)$. An application of Proposition 1 to a suitable affine transform of the vector $\left(M_{n 1}, \ldots, M_{n d}\right)$ then yields the following result. This finding appears in essence in the work of Deheuvels (1978) and Galambos (1987). It is shown in Appendix 2 as a special case of Proposition 3 below, which pertains to triangular arrays.

Proposition 2 Consider a sequence $\left(X_{11}, \ldots, X_{1 d}\right),\left(X_{21}, \ldots, X_{2 d}\right), \ldots$ of $m u$ tually independent random vectors having common distribution function $H$ with margins $F_{1}, \ldots, F_{d}$. Let $H^{*}$ be a multivariate extreme-value distribution with margins $F_{1}^{*}, \ldots, F_{d}^{*}$ and copula $C^{*}$. Then the following statements are equivalent:

(a) $H \in \mathscr{M}\left(H^{*}\right)$.

(b) $F_{j} \in \mathscr{M}\left(F_{j}^{*}\right)$ for $j \in\{1, \ldots, d\}$, and there exists $C \in \mathscr{C}(H)$ such that Eq. 5 holds for all $\left(u_{1}, \ldots, u_{d}\right) \in \operatorname{Ran}\left(F_{1}^{*}\right) \times \cdots \times \operatorname{Ran}\left(F_{d}^{*}\right)=[0,1]^{d}$.

(c) $F_{j} \in \mathscr{M}\left(F_{j}^{*}\right)$ for $j \in\{1, \ldots, d\}$, and Eq. 5 holds uniformly on $[0,1]^{d}$ for all $C \in \mathscr{C}(H)$

Remark 1 Propositions 1 and 2 can be contrasted with the results of Hsing (1989), who does not work with copulas but rather with the unique mapping

$$
B_{H}\left(u_{1}, \ldots, u_{d}\right)=\mathrm{P}\left\{F_{1}\left(X_{1}\right) \leqslant u_{1}, \ldots, F_{d}\left(X_{d}\right) \leqslant u_{d}\right\},
$$

which he calls the "dependence function." His Lemma 3.1 shows that $B_{H_{n}} \rightsquigarrow B_{H}$ as $n \rightarrow \infty$ when $H$ has continuous margins. From his Lemma 2.2 and the proof of his Theorem 3.3, it can also be deduced that if $H \in \mathscr{M}\left(H^{*}\right)$, $B_{H}$ satisfies Eq. 5. 
Note that unless $F_{n 1}, \ldots, F_{n d}$ are continuous, $B_{H_{n}}$ is not a copula. Accordingly, Hsing's results neither imply nor follow from Propositions 1 and 2 , although $B_{H_{n}}$ coincides with every $C \in \mathscr{C}\left(H_{n}\right)$ on $\operatorname{Ran}\left(F_{n 1}\right) \times \cdots \times \operatorname{Ran}\left(F_{n d}\right)$.

At first blush, the uniqueness of $B_{H}$ may seem like an advantage, but it comes at the price of a dependence on the marginal distributions. As a result, range compatibility constraints such as Hsing's condition (3.1) must be introduced and the concept becomes unsuitable for modeling purposes in situations where the marginal distributions are unknown. These issues can be avoided altogether by working with copulas. More importantly, the equivalence between statements (b) and (c) in Propositions 1 and 2 shows that the lack of identifiability of the copula is irrelevant.

Example 2 Let $\left(X_{1}, X_{2}\right)$ be a random pair whose distribution is a bivariate Pareto of the first kind as discussed in Kotz et al. (2000), viz.

$$
H\left(x_{1}, x_{2}\right)=1-\left(\frac{x_{1}}{\theta_{1}}\right)^{-\alpha}-\left(\frac{x_{2}}{\theta_{2}}\right)^{-\alpha}+\left(\frac{x_{1}}{\theta_{1}}+\frac{x_{2}}{\theta_{2}}-1\right)^{-\alpha}=C\left\{F_{1}\left(x_{1}\right), F_{2}\left(x_{2}\right)\right\},
$$

where $\alpha>0$ and $x_{j} \geqslant \theta_{j}>0$ for $j=1,2$. Here, $F_{j}=\mathscr{P}\left(\theta_{j}, \alpha\right), j=1,2$, while the survival copula $\bar{C}\left(u_{1}, u_{2}\right)=u_{1}+u_{2}-1+C\left(1-u_{1}, 1-u_{2}\right)$ is in the Clayton family, i.e.,

$$
\bar{C}\left(u_{1}, u_{2}\right)=\left(u_{1}^{-1 / \alpha}+u_{2}^{-1 / \alpha}-1\right)^{-\alpha} .
$$

Now define the pair $\left(\left\lceil X_{1}\right\rceil,\left\lceil X_{2}\right\rceil\right)$ as in Example 1 and observe that for all $x_{1}, x_{2} \in \mathbb{R}$,

$$
\begin{aligned}
\lceil H\rceil\left(x_{1}, x_{2}\right) & =\mathrm{P}\left(X_{1} \leqslant\left\lfloor x_{1}\right\rfloor, X_{2} \leqslant\left\lfloor x_{2}\right\rfloor\right) \\
& =C\left\{F_{1}\left(\left\lfloor x_{1}\right\rfloor\right), F_{2}\left(\left\lfloor x_{2}\right\rfloor\right)\right\}=C\left\{\left\lceil F_{1}\right\rceil\left(x_{1}\right),\left\lceil F_{2}\right\rceil\left(x_{2}\right)\right\} .
\end{aligned}
$$

In other words, $C \in \mathscr{C}(\lceil H\rceil)$. As argued in Example 1, $\left\lceil F_{1}\right\rceil$ and $\left\lceil F_{2}\right\rceil$ are in the domain of attraction of the Fréchet distribution $\Phi_{\alpha}$. It is also simple to see that Eq. 6 holds with

$$
C^{*}\left(u_{1}, u_{2}\right)=u_{1} u_{2} \exp \left\{\left(\left|\log u_{1}\right|^{-1 / \alpha}+\left|\log u_{2}\right|^{-1 / \alpha}\right)^{-\alpha}\right\},
$$

i.e., the Galambos copula. Thus Proposition 2 implies $\lceil H\rceil \in \mathscr{M}\left(C^{*}\left(\Phi_{\alpha}, \Phi_{\alpha}\right)\right)$.

Example 3 Let $\left(S_{1}, S_{2}\right)$ be a random pair from the Marshall-Olkin bivariate exponential distribution with parameters $\lambda_{0}, \lambda_{1}, \lambda_{2}>0$; see, e.g., Nelsen (2006, Section 3.1.1). For a fixed $c \in(0,1)$, the survival function of the pair $\left(X_{1}, X_{2}\right)=$ $\left(S_{1}^{1 / c}, S_{2}^{1 / c}\right)$ is then

$$
\bar{H}\left(x_{1}, x_{2}\right)=\exp \left\{-\lambda_{1} x_{1}^{c}-\lambda_{2} x_{2}^{c}-\lambda_{0} \max \left(x_{1}^{c}, x_{2}^{c}\right)\right\} .
$$

Hence $F_{j}=\mathscr{W}\left(\lambda_{j}+\lambda_{0}, c\right)$ for $j=1$, 2. Further, $\bar{H}\left(x_{1}, x_{2}\right)=\bar{C}\left\{\bar{F}_{1}\left(x_{1}\right), \bar{F}_{2}\left(x_{2}\right)\right\}$, where

$$
\bar{C}\left(u_{1}, u_{2}\right)=\min \left(u_{1}^{1-\alpha_{1}} u_{2}, u_{1} u_{2}^{1-\alpha_{2}}\right)
$$

is the Marshall-Olkin copula with $\alpha_{j}=\lambda_{0} /\left(\lambda_{j}+\lambda_{0}\right)$ for $j=1,2$. 
Now define $\left(\left\lceil X_{1}\right\rceil,\left\lceil X_{2}\right\rceil\right)$ as in Example 1. As argued there, $\left\lceil F_{1}\right\rceil$ and $\left\lceil F_{2}\right\rceil$ are in the domain of attraction of the Gumbel distribution $\Lambda$. Proceeding as in Example 2, one can establish that $C \in \mathscr{C}(\lceil H\rceil)$. One can also easily see that Eq. 5 or Eq. 6 holds with $C^{*}\left(u_{1}, u_{2}\right)=\Pi\left(u_{1}, u_{2}\right)=u_{1} u_{2}$. Thus Proposition 2 implies $\lceil H\rceil \in \mathscr{M}(\Pi(\Lambda, \Lambda))$.

\section{Extremal behavior of triangular arrays}

For each integer $n \geqslant 1$, let $\left(X_{11: n}, \ldots, X_{1 d: n}\right), \ldots,\left(X_{n 1: n}, \ldots, X_{n d: n}\right)$ be a random sample from a distribution $H_{n}$ with margins $F_{n 1}, \ldots, F_{n d}$. Let

$$
M_{n j}=\max \left(X_{1 j: n}, \ldots, X_{n j: n}\right)
$$

be the componentwise maximum for each $j \in\{1, \ldots, d\}$. The sequence $\left(H_{n}\right)$ is said to belong to the domain of attraction of a non-degenerate distribution $H^{*}$, denoted $\left(H_{n}\right) \in \mathscr{M}\left(H^{*}\right)$, if for every $j \in\{1, \ldots, d\}$, there exist constants $a_{n j}, b_{n j} \in \mathbb{R}$ with $b_{n j}>0$ such that for all continuity points $\left(x_{1}, \ldots, x_{d}\right)$ of $H^{*}$,

$$
\lim _{n \rightarrow \infty} \mathrm{P}\left(\frac{M_{n 1}-a_{n 1}}{b_{n 1}} \leqslant x_{1}, \ldots, \frac{M_{n d}-a_{n d}}{b_{n d}} \leqslant x_{d}\right)=H^{*}\left(x_{1}, \ldots, x_{d}\right) .
$$

It is not generally known whether the limit $H^{*}$ is an extreme-value distribution, but it is clear from Eq. 10 that for each $j \in\{1, \ldots, d\},\left(M_{n j}-a_{n j}\right) / b_{n j}$ converges in law to the margins $F_{j}^{*}$ of $H^{*}$. This may be represented symbolically as follows:

$$
\left(H_{n}\right) \in \mathscr{M}\left(H^{*}\right) \quad \Rightarrow \quad \forall_{j \in\{1, \ldots, d\}} \quad\left(F_{n j}\right) \in \mathscr{M}\left(F_{j}^{*}\right) .
$$

The result below, whose proof is given in Appendix 2, delineates the conditions under which this implication can be reversed. It differs from Proposition 2 in that Eq. 5 is replaced by the weaker statement

$$
\lim _{n \rightarrow \infty} C_{n}^{n}\left(u_{1}^{1 / n}, \ldots, u_{d}^{1 / n}\right)=C^{*}\left(u_{1}, \ldots, u_{d}\right), \quad n \in \mathbb{N}
$$

valid for appropriate choices of $\left(u_{1}, \ldots, u_{d}\right) \in[0,1]^{d}$.

Proposition 3 For each integer $n \geqslant 1$, let $\left(X_{11: n}, \ldots, X_{1: n}\right), \ldots,\left(X_{n 1: n}, \ldots\right.$, $\left.X_{n d: n}\right)$ be a random sample from distribution $H_{n}$ with margins $F_{n 1}, \ldots, F_{n d}$. Let $H^{*}$ be a distribution with margins $F_{1}^{*}, \ldots, F_{d}^{*}$. Then the following statements are equivalent:

(a) $\left(H_{n}\right) \in \mathscr{M}\left(H^{*}\right)$.

(b) $\quad\left(F_{n j}\right) \in \mathscr{M}\left(F_{j}^{*}\right)$ for $j \in\{1, \ldots, d\}$, and there exists a sequence $\left(C_{n}\right)$ of copulas such that $C_{n} \in \mathscr{C}\left(H_{n}\right)$ and Eq. 11 holds for all $\left(u_{1}, \ldots, u_{d}\right) \in$ $\operatorname{Ran}\left(F_{1}^{*}\right) \times \cdots \times \operatorname{Ran}\left(F_{d}^{*}\right)$.

(c) $\left(F_{n j}\right) \in \mathscr{M}\left(F_{j}^{*}\right)$ for $j \in\{1, \ldots, d\}$, and Eq. 11 holds uniformly for all $\left(u_{1}, \ldots, u_{d}\right) \in \overline{\operatorname{Ran}\left(F_{1}^{*}\right)} \times \cdots \times \overline{\operatorname{Ran}\left(F_{d}^{*}\right)}$ for all sequences $\left(C_{n}\right)$ of copulas such that $C_{n} \in \mathscr{C}\left(H_{n}\right)$. 
Remark 2 It can be shown readily that Eq. 11 is equivalent to the statement that for all $x_{1}, \ldots, x_{d} \geqslant 0$ such that $e^{-x_{j}} \in \overline{\operatorname{Ran}\left(F_{j}^{*}\right)}$ for every $j \in\{1, \ldots, d\}$, one has

$$
\lim _{n \rightarrow \infty} \frac{1-C_{n}\left(1-x_{1} / n, \ldots, 1-x_{d} / n\right)}{1 / n}=-\log \left\{C^{*}\left(e^{-x_{1}}, \ldots, e^{-x_{d}}\right)\right\} .
$$

Example 4 For integers $i, k, \ell, n \in \mathbb{N}$ with $1 \leqslant i \leqslant n$, a random pair $\left(X_{i 1: n}, X_{i 2: n}\right)$ is said to follow the geometric distribution of Marshall and Olkin (1985) if

$$
\mathrm{P}\left(X_{i 1: n} \geqslant k, X_{i 2: n} \geqslant \ell\right)=\bar{H}_{n}(k, \ell)= \begin{cases}p_{00 n}^{k} p_{+0 n}^{\ell-k} & \text { if } k<\ell, \\ p_{00 n}^{k} & \text { if } k=\ell, \\ p_{00 n}^{\ell} p_{0+n}^{k-\ell} & \text { if } \ell<k,\end{cases}
$$

in terms of strictly positive parameters $p_{00 n}, p_{01 n}, p_{10 n}$ and $p_{11 n}$ adding up to 1 . Here and in what follows, $p_{r+n}=p_{r 0 n}+p_{r 1 n}$ and $p_{+s n}=p_{0 s n}+p_{1 s n}, r, s \in$ $\{0,1\}$.

Mitov and Nadarajah (2005) show that if $p_{r s n}$ varies with $n$ at an appropriate rate, suitably standardized componentwise maxima $M_{n 1}$ and $M_{n 2}$ from mutually independent random pairs $\left(X_{11: n}, X_{12: n}\right), \ldots,\left(X_{n 1: n}, X_{n 2: n}\right)$ converge jointly to a non-degenerate limit law. In particular, they consider Cases I-IV listed in Table 1 , where $\max \left(p_{1+n}, p_{+1 n}\right) \rightarrow 0$ as $n \rightarrow \infty$. From Nadarajah and Mitov (2002, Corollary 1), $M_{n 1}$ and $M_{n 2}$ then belong to the domain of attraction of the Gumbel distribution, viz.

$$
\lim _{n \rightarrow \infty} \mathrm{P}\left(\frac{M_{n 1}-a_{n 1}}{b_{n 1}} \leqslant t\right)=\lim _{n \rightarrow \infty} \mathrm{P}\left(\frac{M_{n 2}-a_{n 2}}{b_{n 2}} \leqslant t\right)=\exp \left(-e^{-t}\right), \quad t \in \mathbb{R}
$$

where

$$
a_{n 1}=\frac{\log n}{p_{1+n}}, \quad b_{n 1}=\frac{1}{p_{1+n}}, \quad a_{n 2}=\frac{\log n}{p_{+1 n}}, \quad b_{n 2}=\frac{1}{p_{+1 n}} .
$$

Table 1 Four sets of conditions on the parameters of the Marshall-Olkin geometric law

\begin{tabular}{llll}
\hline Case & $p_{11 n}$ & $p_{10 n}$ & $p_{01 n}$ \\
\hline I & $o\left(\frac{1}{\log n}\right)$ & $p_{11 n}\left(\gamma+o\left(\frac{1}{\log n}\right)\right), \gamma \geqslant 0$ & $p_{11 n}\left(\delta+o\left(\frac{1}{\log n}\right)\right), \delta \geqslant 0$ \\
II & $o\left(\frac{p_{01 n}}{\log n}\right)$ & $o\left(\frac{1}{\log n}\right)$ & $\sim p_{10 n}$ \\
III & $o\left(\frac{p_{10 n}}{\log n}\right)$ & $o\left(\frac{1}{\log n}\right)$ & $o\left(\frac{p_{10 n}}{\log n}\right)$ \\
IV & $o\left(\frac{p_{01 n}}{\log n}\right)$ & $o\left(\frac{p_{01 n}}{\log n}\right)$ & $o\left(\frac{1}{\log n}\right)$ \\
\hline
\end{tabular}


Assume that $p_{00 n} \geqslant p_{+0 n} p_{0+n}$ for every integer $n \geqslant 1$ and set

$$
\alpha_{n 1}=1-\frac{\log \left(p_{00 n} / p_{+0 n}\right)}{\log p_{0+n}}, \quad \alpha_{n 2}=1-\frac{\log \left(p_{00 n} / p_{0+n}\right)}{\log p_{+0 n}} .
$$

Then $\alpha_{n j} \in[0,1]$ for $j=1,2$ and it is easily checked that for all $k, \ell \in \mathbb{N}$,

$$
\bar{H}_{n}(k, \ell)=\bar{C}_{n}\left\{\bar{F}_{n 1}(k), \bar{F}_{n 2}(\ell)\right\},
$$

where $F_{n j}$ with $j=1,2$ are the margins of $H_{n}$ while $\bar{C}_{n}$ is the Marshall-Olkin copula with parameters $\alpha_{n j}$ for $j=1,2$, as defined in Eq. 9. Thus $C_{\alpha_{n 1}, \alpha_{n 2}} \in$ $\mathscr{C}\left(H_{n}\right)$ and the result of Mitov and Nadarajah (2005) follows from Proposition 3, provided that Eq. 12 holds for some copula $C^{*}$. Given that for all $x_{1}, x_{2} \geqslant 0$,

$$
\begin{aligned}
\frac{1-C_{n}\left(1-x_{1} / n, 1-x_{2} / n\right)}{1 / n} & =\frac{x_{1} / n+x_{2} / n-\bar{C}_{n}\left(x_{1} / n, x_{2} / n\right)}{1 / n} \\
& =x_{1}+x_{2}-\min \left\{x_{2}\left(x_{1} / n\right)^{1-\alpha_{n 1}}, x_{1}\left(x_{2} / n\right)^{1-\alpha_{n 2}}\right\},
\end{aligned}
$$

it suffices to consider the behavior of $\left(x_{1} / n\right)^{1-\alpha_{n 1}}$ and $\left(x_{2} / n\right)^{1-\alpha_{n 2}}$ as $n \rightarrow \infty$.

For each of the cases in Table 1 , these sequences have to be handled separately, but by symmetry one can restrict to $\left(x_{1} / n\right)^{1-\alpha_{n 1}}$ and to Cases I-III. Further simplification occurs upon noting that

$$
1-\alpha_{n 1}=\frac{\log \left(\frac{1-p_{11 n}-p_{10 n}-p_{01 n}}{1-p_{11 n}-p_{01 n}}\right)}{\log \left(1-p_{11 n}-p_{10 n}\right)}=\frac{\log \left(1-\frac{p_{10 n}}{1-p_{11 n}-p_{01 n}}\right)}{\log \left(1-p_{11 n}-p_{10 n}\right)}=A_{n} \times B_{n},
$$

where

$$
A_{n}=\frac{\log \left(1-\frac{p_{10 n}}{1-p_{11 n}-p_{01 n}}\right)}{p_{10 n} /\left(1-p_{11 n}-p_{01 n}\right)} \times \frac{p_{11 n}+p_{10 n}}{\log \left(1-p_{11 n}-p_{10 n}\right)} \times \frac{1}{1-p_{11 n}-p_{01 n}}
$$

and $B_{n}=p_{10 n} /\left(p_{11 n}+p_{10 n}\right)$. Considering that $\log x \sim x-1$ as $x \rightarrow 1$ and that

$$
\lim _{n \rightarrow \infty} p_{11 n}=\lim _{n \rightarrow \infty} p_{10 n}=\lim _{n \rightarrow \infty} p_{01 n}=0
$$

holds in all cases, one finds that $A_{n} \rightarrow 1$ as $n \rightarrow \infty$. It is also easy to see that

$$
\lim _{n \rightarrow \infty} B_{n}= \begin{cases}\gamma /(1+\gamma) & \text { in Case I, } \\ 1 & \text { in Cases II and III. }\end{cases}
$$

Consequently, $\left(x_{1} / n\right)^{1-\alpha_{n 1}} \rightarrow 0$ as $n \rightarrow \infty$ in Cases II-III, as well as in Case I when $\gamma>0$. When $\gamma=0$ in Case I, however, $B_{n} \log n \rightarrow 0$ as $n \rightarrow \infty$, and hence

$$
\lim _{n \rightarrow \infty}\left(x_{1} / n\right)^{1-\alpha_{n 1}}=\lim _{n \rightarrow \infty} \exp \left\{\left(1-\alpha_{n 1}\right) \log x_{1}-\left(1-\alpha_{n 1}\right) \log n\right\}=1 .
$$

Similarly, one can see that

$$
\lim _{n \rightarrow \infty}\left(x_{2} / n\right)^{1-\alpha_{n 2}}= \begin{cases}1 & \text { in Case I if } \delta=0 \\ 0 & \text { in Case I if } \delta>0 \\ 0 & \text { in Cases II and IV }\end{cases}
$$


The behavior of $\left(x_{1} / n\right)^{1-\alpha_{n 1}}$ and $\left(x_{2} / n\right)^{1-\alpha_{n 2}}$ in Cases IV and III respectively is somewhat more nebulous but of little concern. In Case III, for instance, one has

$$
0 \leqslant \min \left\{x_{2}\left(x_{1} / n\right)^{1-\alpha_{n 1}}, x_{1}\left(x_{2} / n\right)^{1-\alpha_{n 2}}\right\} \leqslant x_{2}\left(x_{1} / n\right)^{1-\alpha_{n 1}},
$$

where the right-hand term vanishes as $n \rightarrow \infty$. In conclusion, $H_{n} \in \mathscr{M}\left(H^{*}\right)$ where

$$
H^{*}\left(x_{1}, x_{2}\right)= \begin{cases}\min \left\{\exp \left(-e^{-x_{1}}\right), \exp \left(-e^{-x_{2}}\right)\right\} & \text { in Case I if } \gamma=\delta=0, \\ \exp \left\{-\left(e^{-x_{1}}+e^{-x_{2}}\right)\right\} & \text { in Case I if } \gamma>0 \text { or } \delta>0, \\ \exp \left\{-\left(e^{-x_{1}}+e^{-x_{2}}\right)\right\} & \text { in Cases II-IV, }\end{cases}
$$

for all $x_{1}, x_{2} \in \mathbb{R}$, as stated by Mitov and Nadarajah (2005).

Example 5 Let $Z_{1}, Z_{2}, Z_{3}$ be mutually independent Poisson random variables and let $\lambda_{i}=\mathrm{E}\left(Z_{i}\right)$ for $i=1,2,3$. Set $X_{j}=Z_{j}+Z_{3}$ for $j=1$,2. The pair $\left(X_{1}, X_{2}\right)$ then has Poisson margins with $\mathrm{E}\left(X_{j}\right)=\lambda_{j}+\lambda_{3}$ for $j=1,2$, and $\operatorname{cov}\left(X_{1}, X_{2}\right)=\lambda_{3}$. Coles and Pauli (2001) consider the extreme-value behavior of a triangular array $\left(X_{11: n}, X_{12: n}\right), \ldots,\left(X_{n 1: n}, X_{n 2: n}\right)$ from this bivariate Poisson distribution with common mean $\mu_{n}$ and covariance $\lambda_{n}$ such that as $n \rightarrow \infty$,

$$
\log n=o\left\{\mu_{n}^{(r+1) /(r+3)}\right\}, \quad\left(1-\frac{\lambda_{n}}{\mu_{n}}\right) \log n \rightarrow \lambda^{2}
$$

for some $r \in \mathbb{N}$ and $\lambda \geqslant 0$. They show that $\left(H_{n}\right) \in \mathscr{M}\left(C_{\lambda}^{*}(\Lambda, \Lambda)\right)$, where $C_{0}^{*}$ is the independence copula and for $\lambda>0, C_{\lambda}^{*}$ is the Hüsler-Reiss copula, viz.

$$
C_{\lambda}^{*}\left(u_{1}, u_{2}\right)=\exp \left[-\tilde{u}_{1} \Phi\left\{\lambda+\frac{1}{2 \lambda} \log \left(\tilde{u}_{1} / \tilde{u}_{2}\right)\right\}-\tilde{u}_{2} \Phi\left\{\lambda+\frac{1}{2 \lambda} \log \left(\tilde{u}_{2} / \tilde{u}_{1}\right)\right\}\right],
$$

with $\tilde{u}_{j}=-\log u_{j}, j=1,2$, and $\Phi$ is the distribution function of the standard normal. Although elements of the class $\mathscr{C}\left(H_{n}\right)$ cannot be written in closed form, Proposition 3 implies that they all satisfy Eq. 11.

\section{Bivariate upper tail dependence}

The bivariate upper tail coefficient of Joe (1993) is a common measure of extremal dependence in a random pair $\left(X_{1}, X_{2}\right)$ with continuous margins $F_{1}$, $F_{2}$. It is defined either as

$$
\lambda\left(X_{1} \mid X_{2}\right)=\lim _{q \rightarrow 1} \mathrm{P}\left\{X_{1}>F_{1}^{-1}(q) \mid X_{2}>F_{2}^{-1}(q)\right\}
$$

or

$$
\lambda\left(X_{2} \mid X_{1}\right)=\lim _{q \rightarrow 1} \mathrm{P}\left\{X_{2}>F_{2}^{-1}(q) \mid X_{1}>F_{1}^{-1}(q)\right\}
$$


and these definitions are equivalent if the limits exist. In the latter case, one can see that if $C$ is the unique copula associated with $\left(X_{1}, X_{2}\right)$, the coefficients coincide with

$$
\lambda(C)=\lim _{q \rightarrow 1} \frac{\bar{C}(1-q, 1-q)}{1-q} .
$$

Moreover, if Eq. 5 holds, it is well known that $\lambda(C)=\lambda\left(C^{*}\right)$; see, e.g., McNeil et al. (2005, Proposition 7.51).

The following proposition extends this result to cases where $F_{1}$ and $F_{2}$ are discontinuous but meet Eq. 7. The proof is given in Appendix 3.

Proposition 4 Let $\left(X_{1}, X_{2}\right)$ be a random pair having joint distribution $H$ with margins $F_{1}, F_{2}$. Assume that $F_{1}$ and $F_{2}$ satisfy Eq. 7 and that $\lambda\left(C_{0}\right)$ exists for some $C_{0} \in \mathscr{C}(H)$. Then the following statements hold:

(a) $\lambda\left(X_{1} \mid X_{2}\right)$ and $\lambda\left(X_{2} \mid X_{1}\right)$ exist.

(b) $\lambda\left(X_{1} \mid X_{2}\right)=\lambda\left(X_{2} \mid X_{1}\right)=\lambda\left(C_{0}\right)$.

(c) For all $C \in \mathscr{C}(H), \lambda(C)$ exists and equals $\lambda\left(C_{0}\right)$.

Now suppose that condition Eq. 7 fails as was the case, e.g., for the triangular arrays in Examples 4 and 5. As illustrated below, it may then happen that neither $\lambda\left(X_{1} \mid X_{2}\right)$ nor $\lambda\left(X_{2} \mid X_{1}\right)$ exists.

Example 6 Let $X_{1}, X_{2}$ be integer-valued random variables with joint distribution $H$ and margins

$$
F_{1}(n)=1-\frac{1}{2^{n}}, \quad F_{2}(n)=\left(1-\frac{1}{2^{n}}\right)^{2}, \quad n \in \mathbb{N} .
$$

In other words, $X_{1}$ is a geometric random variable and $X_{2}$ is from the Lehmann alternative $F_{1}^{2}$. Consider the Gumbel extreme-value copula

$$
C_{\theta}\left(u_{1}, u_{2}\right)=\exp \left\{-\left(\left|\log u_{1}\right|^{\theta}+\left|\log u_{2}\right|^{\theta}\right)^{1 / \theta}\right\}
$$

with parameter $\theta \geqslant 1$. Assume $C_{\theta} \in \mathscr{C}(H)$ for some $\theta>1$. As is well known, $\lambda\left(C_{\theta}\right)=2-2^{1 / \theta}$; see, e.g., McNeil et al. (2005, Example 5.31). Nevertheless, neither $\lambda\left(X_{1} \mid X_{2}\right)$ nor $\lambda\left(X_{2} \mid X_{1}\right)$ exists. The non-existence of $\lambda\left(X_{2} \mid X_{1}\right)$ results from the fact that if $\theta>1$,

$$
\begin{gathered}
\frac{\bar{C}_{\theta}\left\{1-F_{1} \circ F_{1}^{-1}\left(q_{n}\right), 1-F_{2} \circ F_{2}^{-1}\left(q_{n}\right)\right\}}{1-F_{1} \circ F_{1}^{-1}\left(q_{n}\right)} \\
-\frac{\bar{C}_{\theta}\left\{1-F_{1} \circ F_{1}^{-1}\left(r_{n}\right), 1-F_{2} \circ F_{2}^{-1}\left(r_{n}\right)\right\}}{1-F_{1} \circ F_{1}^{-1}\left(r_{n}\right)}
\end{gathered}
$$

does not converge to zero as $n \rightarrow \infty$ for certain choices of sequences $\left(q_{n}\right)$ and $\left(r_{n}\right)$. To be specific, take $q_{n}=F_{1}(n)$ and $r_{n}=F_{2}(n)$ for every $n \in \mathbb{N}$. Observe that $F_{1}(n-1)<F_{2}(n)<F_{1}(n)<F_{2}(n+1)$ and hence for every $n \in \mathbb{N}$,

$$
F_{1}^{-1}\left(q_{n}\right)=F_{1}^{-1}\left(r_{n}\right)=F_{2}^{-1}\left(r_{n}\right)=n \quad \text { and } \quad F_{2}^{-1}\left(q_{n}\right)=n+1 .
$$


In particular, therefore, the expression in Eq. 13 reduces to

$$
\frac{\bar{C}_{\theta}\left\{1-F_{1}(n), 1-F_{2}(n+1)\right\}}{1-F_{1}(n)}-\frac{\bar{C}_{\theta}\left\{1-F_{1}(n), 1-F_{2}(n)\right\}}{1-F_{1}(n)} .
$$

Letting $u_{n 1}=F_{1}(n+1)$ and $u_{n 2}=F_{1}(n)$, one must then consider the limit

$$
\lim _{n \rightarrow \infty} \frac{\bar{C}_{\theta}\left\{2\left(1-u_{n 1}\right), 1-u_{n 1}^{2}\right\}}{2\left(1-u_{n 1}\right)}-\frac{\bar{C}_{\theta}\left(1-u_{n 2}, 1-u_{n 2}^{2}\right)}{1-u_{n 2}} .
$$

That this limit is non-zero stems from the fact that

$$
\lim _{q \rightarrow 1} \frac{\bar{C}_{\theta}\left\{2(1-q), 1-q^{2}\right\}}{2(1-q)}-\lim _{q \rightarrow 1} \frac{\bar{C}_{\theta}\left(1-q, 1-q^{2}\right)}{1-q}=2-2^{1 / \theta}-3+\left(1+2^{\theta}\right)^{1 / \theta},
$$

as can be checked using l'Hospital's rule. The non-existence of $\lambda\left(X_{1} \mid X_{2}\right)$ is shown by analyzing the appropriate limit along the same two sequences.

So long as one of $\lambda\left(X_{1} \mid X_{2}\right)$ or $\lambda\left(X_{2} \mid X_{1}\right)$ exists, however, the upper tail dependence index continues to make sense even when Eq. 7 is not fulfilled. This is the subject of the paper's final proposition, whose proof is in Appendix 4.

Proposition 5 Let $\left(X_{1}, X_{2}\right)$ be a random pair having joint distribution $H$ with margins $F_{1}, F_{2}$ such that $F_{j \rightarrow}\left(x_{j}\right) \rightarrow 1$ as $x_{j} \rightarrow x_{F_{j}}$ for $j=1,2$. Assume that $\lambda\left(C_{0}\right)$ exists for some $C_{0} \in \mathscr{C}(H)$. Then the following statements hold:

(a) If $\lambda\left(X_{1} \mid X_{2}\right)$ exists, then $\lambda\left(X_{1} \mid X_{2}\right)=\lambda(C)$ for all $C \in \mathscr{C}(H)$ such that $\lambda(C)$ exists.

(b) If $\lambda\left(X_{2} \mid X_{1}\right)$ exists, then $\lambda\left(X_{2} \mid X_{1}\right)=\lambda(C)$ for all $C \in \mathscr{C}(H)$ such that $\lambda(C)$ exists.

(c) Both $\lambda\left(X_{1} \mid X_{2}\right)$ and $\lambda\left(X_{2} \mid X_{1}\right)$ exist whenever

$$
\lim _{q \rightarrow 1} \frac{1-F_{1} \circ F_{1}^{-1}(q)}{1-F_{2} \circ F_{2}^{-1}(q)}=1 .
$$

(d) If $\lambda\left(X_{1} \mid X_{2}\right)$ and $\lambda\left(X_{2} \mid X_{1}\right)$ exist and are non-zero, then Eq. 14 holds.

It is reassuring, therefore, that even in the absence of Eq. 7, one always has $\lambda\left(X_{1} \mid X_{2}\right)=\lambda\left(X_{2} \mid X_{1}\right)=\lambda(C)$ whenever the terms exist.

\section{Conclusion}

Extreme-value theory for vectors of discontinuous random variables is still in its infancy. This paper approaches the problem from a copula perspective. In the continuous case, the joint behavior of maxima is known to depend on asymptotic properties of the unique copula associated with the sampling distribution. Proposition 2 extends this result to cases where the underlying 
copula is not necessarily uniquely determined on its entire domain, due to the arbitrary nature of the margins.

As highlighted by Anderson et al. (1997), the extreme-value behavior of discontinuous distributions is often degenerate unless their parameters vary at an appropriate rate as a function of the sample size. For this reason, the limiting behavior of triangular arrays is of practical interest. Proposition 3 shows how it can be characterized in terms of the margins and any compatible copula. This approach can be handy, as in Example 4, which casts new light on the work of Mitov and Nadarajah (2005). It does not work as well when the copula is untractable, however, as illustrated in Example 5.

In recent years, the upper tail dependence coefficient of Joe (1993) has become a widely recognized measure of extremal dependence in bivariate continuous distributions. As shown here, this concept does not generalize readily to the case of arbitrary margins. Unless the marginal distributions meet the condition of Leadbetter et al. (1983), the existence of $\lambda\left(X_{1} \mid X_{2}\right)$ and $\lambda\left(X_{2} \mid X_{1}\right)$ is not guaranteed, even when the variables are connected by a copula $C$ that has a well defined upper tail coefficient. A happy consequence of Proposition 5, however, is that either coefficient coincides with $\lambda(C)$ as soon as it exists.

Acknowledgements Funding in partial support of this work was provided by the Natural Sciences and Engineering Research Council of Canada, the Fonds québécois de la recherche sur la nature et les technologies, the Institut de finance mathématique de Montréal, and the Swiss National Science Foundation.

\section{Appendix 1: Proof of Proposition 1}

It suffices to show that (c) $\Rightarrow$ (b) $\Rightarrow$ (a) $\Rightarrow$ (c). The first implication is trivial. To prove (b) $\Rightarrow$ (a), use the triangle inequality to write

$$
\begin{aligned}
& \left|H_{n}\left(x_{1}, \ldots, x_{d}\right)-H\left(x_{1}, \ldots, x_{d}\right)\right| \\
& \leqslant\left|C_{n}\left\{F_{n 1}\left(x_{1}\right), \ldots, F_{n d}\left(x_{d}\right)\right\}-C_{n}\left\{F_{1}\left(x_{1}\right), \ldots, F_{d}\left(x_{d}\right)\right\}\right| \\
& \quad+\left|C_{n}\left\{F_{1}\left(x_{1}\right), \ldots, F_{d}\left(x_{d}\right)\right\}-C\left\{F_{1}\left(x_{1}\right), \ldots, F_{d}\left(x_{d}\right)\right\}\right|
\end{aligned}
$$

and observe that by hypothesis, the second summand on the right vanishes as $n \rightarrow \infty$. Further note that the first summand is also negligible asymptotically provided that $H$ is continuous at $\left(x_{1}, \ldots, x_{d}\right)$. For, the Lipschitz property of copulas implies that

$$
\left|C_{n}\left\{F_{n 1}\left(x_{1}\right), \ldots, F_{n d}\left(x_{d}\right)\right\}-C_{n}\left\{F_{1}\left(x_{1}\right), \ldots, F\left(x_{d}\right)\right\}\right| \leqslant \sum_{j=1}^{d}\left|F_{n j}\left(x_{j}\right)-F_{j}\left(x_{j}\right)\right|
$$

and the right-hand side can be made arbitrarily small given that $F_{j}$ is continuous at $x_{j}$ for all $j \in\{1, \ldots, d\}$. 
Finally, to see that (a) $\Rightarrow$ (c), first note that the weak convergence of the vector $\left(X_{n 1}, \ldots, X_{n d}\right)$ also applies to the margins by the Continuous Mapping Theorem. Fix $C \in \mathscr{C}(H)$ and an arbitrary sequence $\left(C_{n}\right)$ with $C_{n} \in \mathscr{C}\left(H_{n}\right)$. Let $A$ be the set of vectors $\left(u_{1}, \ldots, u_{d}\right) \in[0,1]^{d}$ such that $u_{j}=F_{j}\left(x_{j}\right)$ for all $j \in\{1, \ldots, d\}$ at some continuity point $\left(x_{1}, \ldots, x_{d}\right)$ of $H$. For arbitrary $\left(u_{1}, \ldots, u_{d}\right) \in A$, one has

$$
\begin{aligned}
& \left|C_{n}\left(u_{1}, \ldots, u_{d}\right)-C\left(u_{1}, \ldots, u_{d}\right)\right| \\
& \quad \leqslant\left|C_{n}\left\{F_{1}\left(x_{1}\right), \ldots, F_{d}\left(x_{d}\right)\right\}-C_{n}\left\{F_{n 1}\left(x_{1}\right), \ldots, F_{n d}\left(x_{d}\right)\right\}\right| \\
& \quad+\left|C_{n}\left\{F_{n 1}\left(x_{1}\right), \ldots, F_{n d}\left(x_{d}\right)\right\}-C\left\{F_{1}\left(x_{1}\right), \ldots, F_{d}\left(x_{d}\right)\right\}\right| .
\end{aligned}
$$

Now as $n \rightarrow \infty$, the first summand on the right is negligible by the inequality in Eq. 15 while the second summand is $\left|H_{n}\left(x_{1}, \ldots, x_{d}\right)-H\left(x_{1}, \ldots, x_{d}\right)\right|$ and tends to zero by hypothesis. Consequently, $C_{n} \rightarrow C$ on $A$, which is dense in $\overline{\operatorname{Ran}\left(F_{1}\right)} \times \cdots \times \overline{\operatorname{Ran}\left(F_{d}\right)}$. As the sequence $\left(C_{n}\right)$ is equicontinuous because of the Lipschitz property of copulas, the convergence immediately extends to the latter set, and uniform convergence follows from the Arzelà-Ascoli Theorem.

\section{Appendix 2: Proofs of Propositions 2-3}

Both proofs rely on the fact that if $C$ is a copula, then so is the mapping $D_{n}$ defined by Eq. 8. This comes from the fact that if $\left(U_{11}, \ldots, U_{1 d}\right), \ldots,\left(U_{n 1}, \ldots, U_{n d}\right)$ is a random sample from $C$, the joint distribution of the componentwise maxima $M_{n j}=\max \left(U_{1 j}, \ldots, U_{n j}\right)$ for $j \in$ $\{1, \ldots, d\}$ can be expressed in the form

$$
\mathrm{P}\left(M_{n 1} \leqslant u_{1}, \ldots, M_{n d} \leqslant u_{d}\right)=C^{n}\left(u_{1}, \ldots, u_{d}\right)=D_{n}\left(u_{1}^{n}, \ldots, u_{d}^{n}\right),
$$

where $u_{j}^{n}=\mathrm{P}\left(M_{n j} \leqslant u_{j}\right)$ for $j \in\{1, \ldots, d\}$.

As Proposition 2 derives from Proposition 3, the latter is established first. It is sufficient to show that (c) $\Rightarrow$ (b) $\Rightarrow$ (a) $\Rightarrow$ (c), and the first implication is trivial. Given constants $a_{n j}, b_{n j} \in \mathbb{R}$ with $b_{n j}>0$, introduce the distribution function

$$
\tilde{H}_{n}\left(x_{1}, \ldots, x_{d}\right)=H_{n}^{n}\left(a_{n 1}+b_{n 1} x_{1}, \ldots, a_{n d}+b_{n d} x_{d}\right),
$$

with marginals $\tilde{F}_{n 1}, \ldots, \tilde{F}_{n d}$. Note that if $C_{n} \in \mathscr{C}\left(H_{n}\right)$, then for all $x_{1}, \ldots$, $x_{d} \in \mathbb{R}$,

$$
\begin{aligned}
\mathrm{P}\left(M_{n 1}\right. & \left.\leqslant a_{n 1}+b_{n 1} x_{1}, \ldots, M_{n d} \leqslant a_{n d}+b_{n d} x_{d}\right) \\
& =\tilde{H}_{n}\left(x_{1}, \ldots, x_{d}\right)=C_{n}^{n}\left\{\tilde{F}_{n 1}^{1 / n}\left(x_{1}\right), \ldots, \tilde{F}_{n d}^{1 / n}\left(x_{d}\right)\right\} \\
& =D_{n}\left\{\tilde{F}_{n 1}\left(x_{1}\right), \ldots, \tilde{F}_{n d}\left(x_{d}\right)\right\} .
\end{aligned}
$$

Thus $C_{n} \in \mathscr{C}\left(H_{n}\right) \Rightarrow D_{n} \in \mathscr{C}\left(\tilde{H}_{n}\right)$.

To prove (b) $\Rightarrow$ (a), suppose that for each $j \in\{1, \ldots, d\}, a_{n j}$ and $b_{n j}>0$ are constants for which $\tilde{F}_{n j} \rightsquigarrow F_{j}^{*}$ as $n \rightarrow \infty$. Further assume that $\left(C_{n}\right)$ is a se- 
quence for which Eq. 11 holds for all $\left(u_{1}, \ldots, u_{d}\right) \in \overline{\operatorname{Ran}\left(F_{1}^{*}\right)} \times \cdots \times \overline{\operatorname{Ran}\left(F_{d}^{*}\right)}$. Then $D_{n} \rightarrow C^{*}$ on the same domain, and hence by Proposition 1, $\tilde{H}_{n} \rightsquigarrow H^{*}$ as $n \rightarrow \infty$.

Finally, to see that (a) $\Rightarrow$ (c), suppose that $\left(H_{n}\right) \in \mathscr{M}\left(H^{*}\right)$, i.e., $\tilde{H}_{n} \rightsquigarrow H^{*}$ as $n \rightarrow \infty$. It is then immediate that $\tilde{F}_{n j} \rightsquigarrow F_{j}^{*}$ for all $j \in\{1, \ldots, d\}$. Now if $\left(E_{n}\right)$ is an arbitrary sequence of copulas such that $E_{n} \in \mathscr{C}\left(\tilde{H}_{n}\right)$ and if $C^{*}$ is an arbitrary element of $\mathscr{C}\left(H^{*}\right)$, then by Proposition $1, E_{n} \rightarrow C^{*}$ uniformly on $\overline{\operatorname{Ran}\left(F_{1}^{*}\right)} \times \cdots \times \overline{\operatorname{Ran}\left(F_{d}^{*}\right)}$. In particular, if $C \in \mathscr{C}\left(H_{n}\right)$ and $D_{n}$ is defined by Eq. 8, one concludes that $D_{n} \rightarrow C^{*}$ uniformly on the same domain, as claimed.

Proposition 2 follows readily from Proposition 3 upon setting $H_{n}=H$ for all $n \geqslant 1$. In that special case, Eq. 11 reduces to

$$
\lim _{n \rightarrow \infty} C^{n}\left(u_{1}^{1 / n}, \ldots, u_{d}^{1 / n}\right)=C^{*}\left(u_{1}, \ldots, u_{d}\right), \quad n \in \mathbb{N} .
$$

The latter is equivalent to Eq. 5 in view of the fact that for all $t>0$,

$$
\begin{aligned}
C^{\lfloor t\rfloor+1}\left(u_{1}^{1 /\lfloor t\rfloor}, \ldots, u_{d}^{1 /\lfloor t\rfloor}\right) & \leqslant C^{t}\left(u_{1}^{1 / t}, \ldots, u_{d}^{1 / t}\right) \\
& \leqslant C^{\lfloor t\rfloor}\left(u_{1}^{1 /(\lfloor t\rfloor+1)}, \ldots, u_{d}^{1 /(\lfloor t\rfloor+1)}\right) .
\end{aligned}
$$

\section{Appendix 3: Proof of Proposition 4}

First note that for $j=1,2$ and all $q \in(0,1)$, one has

$$
1-F_{j} \circ F_{j}^{-1}(q) \leqslant 1-q \leqslant 1-F_{j \rightarrow \circ} \circ F_{j}^{-1}(q) .
$$

Thus

$$
\lim _{q \rightarrow 1} \frac{1-F_{j} \circ F_{j}^{-1}(q)}{1-F_{j \rightarrow} \circ F_{j}^{-1}(q)} \leqslant \lim _{q \rightarrow 1} \frac{1-F_{j} \circ F_{j}^{-1}(q)}{1-q} \leqslant 1,
$$

so that because $F_{j}$ satisfies Eq. 7 by hypothesis,

$$
\lim _{q \rightarrow 1} \frac{1-F_{j} \circ F_{j}^{-1}(q)}{1-q}=1
$$

for $j=1,2$. Consequently, Eq. 14 holds. Proposition 5 then implies that $\lambda\left(X_{1} \mid X_{2}\right)$ and $\lambda\left(X_{2} \mid X_{1}\right)$ both exist, and that $\lambda\left(X_{1} \mid X_{2}\right)=\lambda\left(X_{2} \mid X_{1}\right)=\lambda(C)$ for every $C \in \mathscr{C}(H)$ for which the latter index exists. This shows parts (a) and (b) of Proposition 4. 
To prove part (c), pick an arbitrary $C \in \mathscr{C}(H)$. It then follows from the inequalities in Eq. 16 that

$$
\begin{aligned}
& \frac{\bar{C}\left\{1-F_{1} \circ F_{1}^{-1}(q), 1-F_{2} \circ F_{2}^{-1}(q)\right\}}{1-q} \\
& \leqslant \frac{\bar{C}(1-q, 1-q)}{1-q} \\
& \leqslant \frac{\bar{C}\left\{1-F_{1 \rightarrow} \circ F_{1}^{-1}(q), 1-F_{2 \rightarrow} \circ F_{2}^{-1}(q)\right\}}{1-q} .
\end{aligned}
$$

Observe that

$$
\begin{aligned}
& \lim _{q \rightarrow 1} \frac{\bar{C}\left\{1-F_{1} \circ F_{1}^{-1}(q), 1-F_{2} \circ F_{2}^{-1}(q)\right\}}{1-q} \\
& \quad=\lim _{q \rightarrow 1} \frac{\bar{C}\left\{1-F_{1} \circ F_{1}^{-1}(q), 1-F_{2} \circ F_{2}^{-1}(q)\right\}}{1-F_{1} \circ F_{1}^{-1}(q)} \times \frac{1-F_{1} \circ F_{1}^{-1}(q)}{1-q}=\lambda\left(C_{0}\right)
\end{aligned}
$$

by parts (a) and (b), as well as the fact that Eq. 7 holds for $F_{1}$. Now define

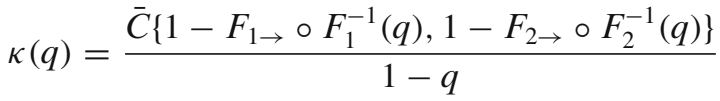

$$
\begin{aligned}
& -\frac{\bar{C}\left\{1-F_{1} \circ F_{1}^{-1}(q), 1-F_{2} \circ F_{2}^{-1}(q)\right\}}{1-q}
\end{aligned}
$$

and invoke the Lipschitz property of copulas to deduce that

$$
\begin{aligned}
|\kappa(q)| \leqslant & \frac{\left|F_{1} \circ F_{1}^{-1}(q)-F_{1 \rightarrow} \circ F_{1}^{-1}(q)\right|}{1-F_{1} \circ F_{1}^{-1}(q)} \times \frac{1-F_{1} \circ F_{1}^{-1}(q)}{1-q} \\
& +\frac{\left|F_{2} \circ F_{2}^{-1}(q)-F_{2 \rightarrow} \circ F_{2}^{-1}(q)\right|}{1-F_{2} \circ F_{2}^{-1}(q)} \times \frac{1-F_{2} \circ F_{2}^{-1}(q)}{1-q} .
\end{aligned}
$$

In view of Eqs. 7 and 17, $\kappa(q) \rightarrow 0$ as $q \rightarrow 1$. Thus, $\lambda(C)$ exists and equals $\lambda\left(C_{0}\right)$.

\section{Appendix 4: Proof of Proposition 5}

The proof relies on the fact that

$$
r_{I}=\liminf _{q \rightarrow 1} \frac{1-F_{1} \circ F_{1}^{-1}(q)}{1-F_{2} \circ F_{2}^{-1}(q)} \leqslant 1 \leqslant \limsup _{q \rightarrow 1} \frac{1-F_{1} \circ F_{1}^{-1}(q)}{1-F_{2} \circ F_{2}^{-1}(q)}=r_{S} .
$$

To show the first inequality, call on the relations in Eq. 16 to write

$$
r_{I} \leqslant \liminf _{q \rightarrow 1} \frac{1-q}{1-F_{2} \circ F_{2}^{-1}(q)},
$$


and observe that the right-hand term is at most 1 . To see this, use the fact that $F_{2 \rightarrow}\left(x_{2}\right) \rightarrow 1$ as $x_{2} \rightarrow x_{F_{2}}$ to construct a sequence $\left(t_{n}\right)$ such that $F_{2}\left(t_{n}\right)=$ $q_{n} \rightarrow 1$ as $n \rightarrow \infty$. For such a sequence, $\left(1-q_{n}\right) /\left\{1-F_{2} \circ F_{2}^{-1}\left(q_{n}\right)\right\}=1$ for all $n \in \mathbb{N}$, and hence $r_{I} \leqslant 1$. The inequality $r_{S} \geqslant 1$ can be shown analogously.

Statements (a) and (b) are identical, except for a change of indices. To establish the former, first write

$$
\begin{aligned}
\lambda\left(X_{1} \mid X_{2}\right) & =\lim _{q \rightarrow 1} \frac{\bar{C}_{0}\left\{1-F_{1} \circ F_{1}^{-1}(q), 1-F_{2} \circ F_{2}^{-1}(q)\right\}}{1-F_{2} \circ F_{2}^{-1}(q)} \\
& =\lim _{q \rightarrow 1} \frac{\bar{C}_{0}\left[R(q)\left\{1-F_{2} \circ F_{2}^{-1}(q)\right\}, 1-F_{2} \circ F_{2}^{-1}(q)\right]}{1-F_{2} \circ F_{2}^{-1}(q)},
\end{aligned}
$$

where $R(q)=\left\{1-F_{1} \circ F_{1}^{-1}(q)\right\} /\left\{1-F_{2} \circ F_{2}^{-1}(q)\right\}$. Next, let $\mathscr{R}$ be the set of reals $r$ for which $R\left(q_{n}\right) \rightarrow r$ for some sequence $\left(q_{n}\right)$ in $(0,1)$ such that $q_{n} \rightarrow 1$ as $n \rightarrow \infty$. Then for all $r \in \mathscr{R}$,

$$
\lambda\left(X_{1} \mid X_{2}\right)=\lim _{n \rightarrow \infty} \frac{\bar{C}_{0}\left[r\left\{1-F_{2} \circ F_{2}^{-1}\left(q_{n}\right)\right\}, 1-F_{2} \circ F_{2}^{-1}\left(q_{n}\right)\right]}{1-F_{2} \circ F_{2}^{-1}\left(q_{n}\right)} .
$$

To show this, introduce

$$
\begin{aligned}
\delta(n)= & \frac{\bar{C}_{0}\left[r\left\{1-F_{2} \circ F_{2}^{-1}\left(q_{n}\right)\right\}, 1-F_{2} \circ F_{2}^{-1}\left(q_{n}\right)\right]}{1-F_{2} \circ F_{2}^{-1}\left(q_{n}\right)} \\
& -\frac{\bar{C}_{0}\left[R\left(q_{n}\right)\left\{1-F_{2} \circ F_{2}^{-1}\left(q_{n}\right)\right\}, 1-F_{2} \circ F_{2}^{-1}\left(q_{n}\right)\right]}{1-F_{2} \circ F_{2}^{-1}\left(q_{n}\right)}
\end{aligned}
$$

and observe that $|\delta(n)| \leqslant\left|R\left(q_{n}\right)-r\right| \rightarrow 0$ as $n \rightarrow \infty$ by the Lipschitz property of copulas. Thus if $1 \in \mathscr{R}, \lambda\left(X_{1} \mid X_{2}\right)=\lambda\left(C_{0}\right)$. Otherwise the inequalities in Eq. 18 are strict. In that case, pick a sequence $\left(q_{n}\right)$ in $(0,1)$ such that $q_{n} \rightarrow 1$ and $R\left(q_{n}\right) \rightarrow r_{I}$ as $n \rightarrow \infty$. Then by Eq. 19 and the monotonicity of $\bar{C}_{0}$, one has

$$
\begin{aligned}
\lambda\left(X_{1} \mid X_{2}\right) & =\lim _{n \rightarrow \infty} \frac{\bar{C}_{0}\left[r_{I}\left\{1-F_{2} \circ F_{2}^{-1}\left(q_{n}\right)\right\}, 1-F_{2} \circ F_{2}^{-1}\left(q_{n}\right)\right]}{1-F_{2} \circ F_{2}^{-1}\left(q_{n}\right)} \\
& \leqslant \lim _{n \rightarrow \infty} \frac{\bar{C}_{0}\left\{1-F_{2} \circ F_{2}^{-1}\left(q_{n}\right), 1-F_{2} \circ F_{2}^{-1}\left(q_{n}\right)\right\}}{1-F_{2} \circ F_{2}^{-1}\left(q_{n}\right)}=\lambda\left(C_{0}\right) .
\end{aligned}
$$

Similarly, one can show that $\lambda\left(X_{1} \mid X_{2}\right) \geqslant \lambda\left(C_{0}\right)$ by considering the limit along a sequence $\left(q_{n}\right)$ such that $R\left(q_{n}\right) \rightarrow r_{S}$ as $n \rightarrow \infty$. Thus, $\lambda\left(X_{1} \mid X_{2}\right)=\lambda\left(C_{0}\right)$ as claimed.

To prove (c), take $C_{0} \in \mathscr{C}(H)$ such that $\lambda\left(C_{0}\right)$ exists and introduce

$$
\begin{aligned}
\gamma(q)= & \frac{\bar{C}_{0}\left\{1-F_{1} \circ F_{1}^{-1}(q), 1-F_{2} \circ F_{2}^{-1}(q)\right\}}{1-F_{2} \circ F_{2}^{-1}(q)} \\
& -\frac{\bar{C}_{0}\left\{1-F_{2} \circ F_{2}^{-1}(q), 1-F_{2} \circ F_{2}^{-1}(q)\right\}}{1-F_{2} \circ F_{2}^{-1}(q)} .
\end{aligned}
$$


Note that from the Lipschitz property of copulas,

$$
|\gamma(q)| \leqslant \frac{\left|F_{1} \circ F_{1}^{-1}(q)-F_{2} \circ F_{2}^{-1}(q)\right|}{1-F_{2} \circ F_{2}^{-1}(q)} \rightarrow 0
$$

as $q \rightarrow 1$ whenever Eq. 14 holds. Taking limits on both sides of Eq. 20, one sees from its definition that $\lambda\left(X_{1} \mid X_{2}\right)$ exists and equals $\lambda\left(C_{0}\right)$. Similarly, $\lambda\left(X_{2} \mid X_{1}\right)$ is well defined and coincides with $\lambda\left(C_{0}\right)$.

Finally, to get (d), suppose that $\lambda\left(X_{1} \mid X_{2}\right)$ and $\lambda\left(X_{2} \mid X_{1}\right)$ exist and are nonzero. Then

$$
\begin{aligned}
\lambda\left(X_{1} \mid X_{2}\right) & =\lim _{q \rightarrow 1} \frac{\mathrm{P}\left\{X_{1}>F_{1}^{-1}(q), X_{2}>F_{2}^{-1}(q)\right\}}{1-F_{2} \circ F_{2}^{-1}(q)} \\
& =\lim _{q \rightarrow 1} \frac{\mathrm{P}\left\{X_{1}>F_{1}^{-1}(q), X_{2}>F_{2}^{-1}(q)\right\}}{1-F_{1} \circ F_{1}^{-1}(q)} \times \frac{1-F_{1} \circ F_{1}^{-1}(q)}{1-F_{2} \circ F_{2}^{-1}(q)} \\
& =\lambda\left(X_{2} \mid X_{1}\right) \times \lim _{q \rightarrow 1} \frac{1-F_{1} \circ F_{1}^{-1}(q)}{1-F_{2} \circ F_{2}^{-1}(q)},
\end{aligned}
$$

which shows that the limit in Eq. 14 exists. However, by Eq. 18, the latter equals 1 .

\section{References}

Anderson, C.W., Coles, S.G., Hüsler, J.: Maxima of Poisson-like variables and related triangular arrays. Ann. Appl. Probab. 7, 953-971 (1997)

Beirlant, J., Goegebeur, Y., Teugels, J.L., Segers, J.: Statistics of Extremes. Wiley, Chichester (2004)

Coles, S.G., Pauli, F.: Extremal limit laws for a class of bivariate Poisson vectors. Stat. Probab. Lett. 54, 373-379 (2001)

Deheuvels, P.: Caractérisation complète des lois extrêmes multivariées et de la convergence aux types extrêmes. Publ. Inst. Stat. Univ. Paris 22, 1-36 (1978)

Embrechts, P., Klüppelberg, C., Mikosch, T.: Modelling Extremal Events for Insurance and Finance. Springer, New York (1997)

Fisher, R.A., Tippett, L.H.C.: Limiting forms of the frequency distributions of the largest or smallest member of a sample. Proc. Camb. Philos. Soc. 24, 180-190 (1928)

Fougères, A.-L.: Multivariate extremes. In: Finkenstädt, B., Rootzén, H. (eds.) Extreme Values in Finance, Telecommunications, and the Environment, Monographs on Statistics and Applied Probability 99. Chapman \& Hall, London (2004)

Galambos, J.: The Asymptotic Theory of Extreme Order Statistics, 2nd edn. Robert E. Krieger Publishing Co. Inc., Melbourne, FL (1987)

Genest, C., Nešlehová, J.: A primer on copulas for count data. ASTIN Bull. 37, 475-515 (2007)

Hsing, T.: Extreme value theory for multivariate stationary sequences. J. Multivar. Anal. 29, 274-291 (1989)

Joe, H.: Multivariate dependence measures and data analysis. Comput. Stat. Data Anal. 16, 279-297 (1993)

Kotz, S., Balakrishnan, N., Johnson, N.L.: Continuous Multivariate Distributions, 2nd edn. Wiley, New York (2000)

Leadbetter, M.R., Lindgren, G., Rootzén, H.: Extremes and Related Properties of Random Sequences and Processes. Springer, New York (1983) 
Marshall, A.W.: Copulas, marginals, and joint distributions. In: Distributions with Fixed Marginals and Related Topics (Seattle, WA, 1993). IMS Lecture Notes Monogr. Ser., vol. 28, pp. 213-222. Inst. Math. Statist., Hayward, CA (1996)

Marshall, A.W., Olkin, I.: A family of bivariate distributions generated by the bivariate Bernoulli distribution. J. Am. Stat. Assoc. 80, 332-338 (1985)

McNeil, A.J., Frey, R., Embrechts, P.: Quantitative Risk Management: Concepts, Techniques, and Tools. Princeton University Press, Princeton, NJ (2005)

Mitov, K., Nadarajah, S.: Limit distributions for the bivariate geometric maxima. Extremes 8, $357-$ $370(2005)$

Mitov, K.V., Pakes, A.G., Yanev, G.P.: Extremes of geometric variables with applications to branching processes. Stat. Probab. Lett. 65, 379-388 (2003)

Nadarajah, S., Mitov, K.: Asymptotics of maxima of discrete random variables. Extremes 5, 287-294 (2002)

Nadarajah, S., Mitov, K.: Extremal limit laws for discrete random variables. J. Math. Sci. (N.Y.) 122, 3404-3415 (2004)

Nelsen, R.B.: An Introduction to Copulas, 2nd edn. Springer, New York (2006)

Resnick, S.I.: Extreme Values, Regular Variation, and Point Processes. Springer, New York (1987) 